\title{
Statistical Method for Semantic Segmentation of Dominant Plane from Remote Exploration Image Sequence
}

\author{
Shun Inagaki ${ }^{1}$ and Atsushi Imiya ${ }^{2}$ \\ 1 School of Advanced Integration Science, Chiba University \\ 2 Institute of Management and Information Technologies, Chiba University \\ Yayoicho 1-33, Inage-ku, Chiba, 263-8522, Japan
}

\begin{abstract}
For the application of well-established image analysis algorithms to low frame-rate image sequences, which are common in bioimaging and long-distance extrapolation, we are required to up-convert the frame-rate of image sequences. For the motion analysis of low framerate image sequences, we introduce a method for semantic segmentation of the dominant plane, which is the largest planar area on an image plane, from a low frame-rate image sequence, which is common for image sequence obtained by remote extrapolation.
\end{abstract}

\section{Introduction}

In this paper, we introduce a method for semantic segmentation of the dominant plane from the optical flow field of a low frame-rate image sequence combining image registration [5] and optical flow computation [8, 7].

The optical flow field is a fundamental feature for the interpretation of temporal image sequences [6]. For the motion analysis from low frame-rate image sequence, we are required to generate a dense optical flow fields, since wellestablished algorithms for motion analysis do not assume the use of low framerate image sequences.

For the detection of safe areas for navigation, the robot probe detects the dominant plane, which is the largest planar area on an image plane, from a sequence of images captured by a camera mounted on the robot. In the image, the safe areas and obstacle areas for navigation are detected using the optical flow field and homography of the ground plane [2 [4]. Figure1 (a) shows a cycle for autonomous navigation using optical flow.

Using an uncalibrated monocular camera as a sensor for obtaining information on the environment, in ref. [1], a featureless robot navigation method based on a planar area and an optical flow field computed from a pair of successive images is proposed. A planar area in an environment is called a dominant plane, and it corresponds to the largest part of an image. We accept the following five assumptions. 


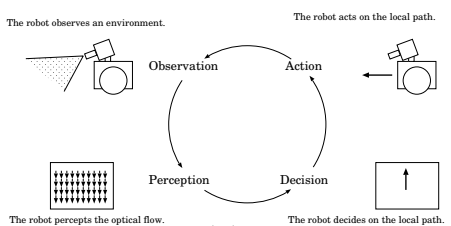

(a)

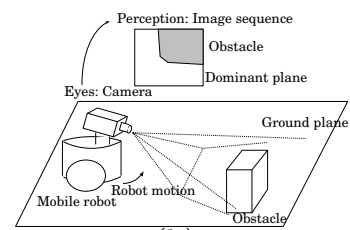

(b)

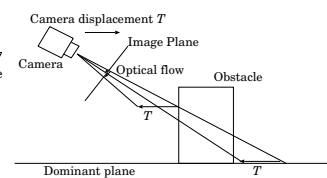

(c)

Fig. 1. Observation-Perception-Decision-Action cycle for vision-based robot navigation. (a) First, a mobile robot equipped with a camera observes the environment. Next, an optical flow field relative to the robot motion is computed from images obtained by the camera. The optical flow field is used to decide the local path. (b) The mobile robot has a camera, which corresponds to its eyes. The robot perceives an optical flow field from its ego-motion. (c) If the camera moves a distance $T$ approximately parallel to the dominant plane, the optical flow vectors on the obstacle and on the dominant plane areas have the same distance $T$. However, they differ at the same time.

1. The ground plane is the planar area.

2. The camera mounted on the mobile robot is looking downward.

3. The robot observes the environment using the camera mounted on itself for navigation.

4. The camera on the robot captures a sequence of images while the robot is moving.

5. The planar area occupies more than $1 / 2$ on the image.

These assumptions are illustrated in Figs. 1 (b) and (c).

Since the planar flow vector on the ground plane is equal to the optical flow vector $\dot{\boldsymbol{x}}$ on the dominant plane, we use the difference between these two flows to detect the dominant plane. From the assumption 1 and 2, we have the following property.

Property 1. Corresponding points on a dominant plane in a pair of successive images are combined by homography.

\section{Dominant Plane and Optical Flow}

Setting $\boldsymbol{H}$ to be a $3 \times 3$ matrix [14], the homography between two images of a planar surface can be expressed as $\boldsymbol{\xi}^{\prime}=\boldsymbol{H} \boldsymbol{\xi}$, where $\boldsymbol{\xi}=(x, y, 1)^{\top}$ and $\boldsymbol{\xi}^{\prime}=\left(x^{\prime}, y^{\prime}, 1\right)^{\top}$ are the homogeneous coordinates of corresponding points in two successive images. Assuming that the camera displacement is small, the matrix $\boldsymbol{H}$ can be approximated by affine transformations. These geometrical and mathematical assumptions are valid when the camera is mounted on a mobile robot moving on the dominant plane. Therefore, the corresponding points $\boldsymbol{x}=(x, y)^{\top}$ and $\boldsymbol{x}^{\prime}=\left(x^{\prime}, y^{\prime}\right)^{\top}$ on the dominant plane are related by $\boldsymbol{x}^{\prime}=\boldsymbol{A} \boldsymbol{x}+\boldsymbol{b}$, where $\boldsymbol{A}$ and $\boldsymbol{b}$ are a $2 \times 2$ affine-coefficient matrix and a two-dimensional vector, respectively, which are approximations of $\boldsymbol{H}$. This geometric relation implies that $\boldsymbol{u}(x, y, t+1)=\boldsymbol{A} \boldsymbol{x}+\boldsymbol{b}-\boldsymbol{x}$ and that the next property is satisfied. 
Property 2. On the dominant plane, the optical flow vector is stationary and the planar-flow vector on the ground plane is equal to the optical flow vector $\dot{\boldsymbol{x}}=\boldsymbol{u}$.

We call $\boldsymbol{x}^{\prime}-\boldsymbol{x}$ the planar flow. The RANSAC-based method [11] for the estimation of the affine coefficients is described as Algorithm 1.

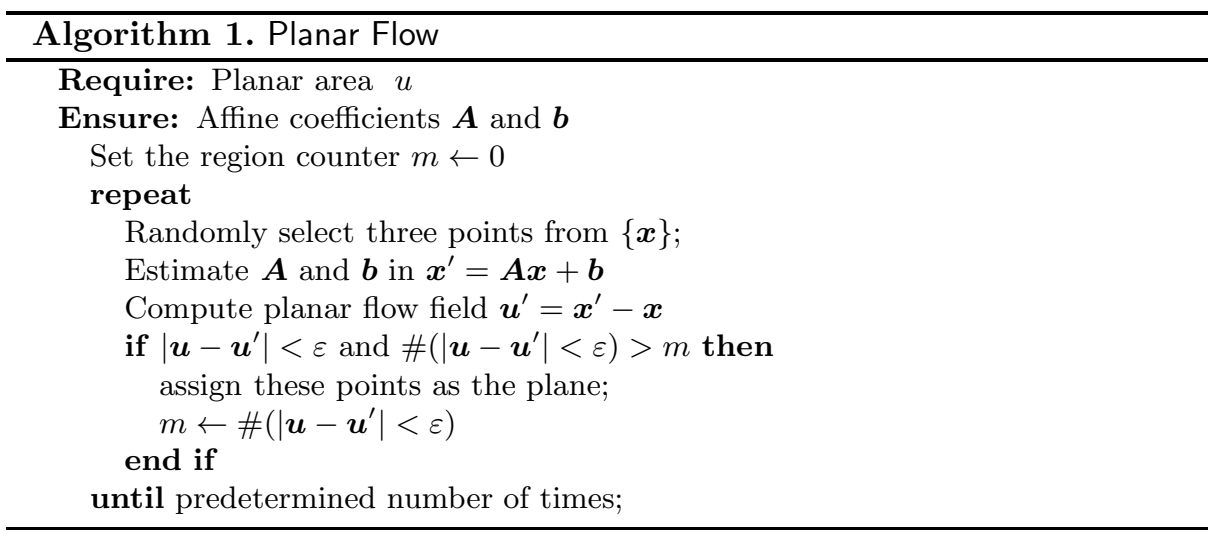

Once the affine coefficients are estimated, we can extract a segment for the dominant plane. We use the difference between these two flows for semantic segmentation of the dominant plane. Therefore, if $|\boldsymbol{u}(x, y, t)-\boldsymbol{u}(x, y, t+1)|<\epsilon$ for a small positive number $\epsilon$, we conclude that the point $\boldsymbol{x}=(x, y)^{\top}$ lies on the dominant plane. If an obstacle exists in front of the robot, the planar flow on the image plane differs from the optical flow on the image plane as shown in Fig. 1 (c).

\section{Semantic Segmentation Using Subframe Motion}

We develop an algorithm to compute the optical flow field of a temporal image sequence $f\left(\boldsymbol{x}, t+\frac{1}{2}\right)$, in which we set $\boldsymbol{u}_{\frac{1}{2}}(\boldsymbol{x}, t)$ from $f(\boldsymbol{x}, t)$ and $f(\boldsymbol{x}, t+1)$. For the convenience of analysis, we set

$$
\begin{aligned}
& f^{+}(\boldsymbol{x})=f(\boldsymbol{x}, t+1), f^{-}(\boldsymbol{x}, t)=f(\boldsymbol{x}, t), g(\boldsymbol{x})=f\left(\boldsymbol{x}, t+\frac{1}{2}\right), \\
& \boldsymbol{v}=\boldsymbol{u}_{\frac{1}{2}}(\boldsymbol{x}, t), \quad \boldsymbol{w}=\boldsymbol{u}_{\frac{1}{2}}\left(\boldsymbol{x}, t+\frac{1}{2}\right) .
\end{aligned}
$$

Algorithm 2 shows the procedure for dominant plane detection using subframe optical flow computation. 


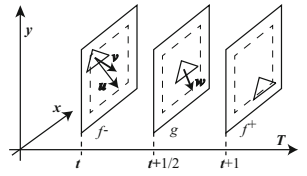

(a) Geometrical relation

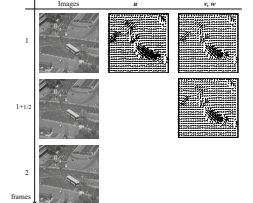

(b) Ettlinger-Tor

Fig. 2. Geometrical relations among images sequence and computational results. (a) Geometrical relations among $g, f^{-}, f^{+}, \boldsymbol{u}, \boldsymbol{v}$ and $\boldsymbol{w}$. (b) Computational results the original and subframe optical flow fields for the Etllinger-Tor sequence.

For large-displacement image sequences, we compute the dominant plane from $\boldsymbol{v}$ and $\boldsymbol{w}$, which are computed from $f(x, y, t)$ and $f(x, y, t+1 / 2)$, and from $f(x, y, t+1 / 2)$ and $f(x, y, t+1)$, respectively, using the subframe optical flow computation method derived in the previous section. We conclude that the point $\boldsymbol{x}=(x, y)^{\top}$ lies on the dominant plane if $|\boldsymbol{u}-\boldsymbol{w}|<\epsilon$.

For $f(\boldsymbol{x}, t)$ and $f(\boldsymbol{x}, t+1)$, if the relation

$$
\nabla f(\boldsymbol{x}, t) \cong \nabla f\left(\boldsymbol{x}, t+\frac{1}{2}\right)=\nabla f(\boldsymbol{x}, t)+\delta(\boldsymbol{x})
$$

is satisfied, we have the next theorem.

Theorem 1. The relation $\nabla f^{\top}(\boldsymbol{v}+\boldsymbol{w})+f_{t} \cong 0$, where $\nabla f(\boldsymbol{x}, t)^{\top} \boldsymbol{v}+f_{t}(\boldsymbol{x}, t)=0$ and $\nabla f\left(\boldsymbol{x}, t+\frac{1}{2}\right)^{\top} \boldsymbol{w}+f_{t}\left(\boldsymbol{x}, t+\frac{1}{2}\right)=0$, is satisfied 1 .

Setting $g(\boldsymbol{x})=f^{+}(\boldsymbol{x}-\boldsymbol{w}), g(\boldsymbol{x})=f^{-}(\boldsymbol{x}+\boldsymbol{v})$ and $\boldsymbol{u}=\boldsymbol{v}+\boldsymbol{w}$, Theorem 1 implies that we can have $g, \boldsymbol{v}$ and $\boldsymbol{w}$ as the minimisers of

$$
J(g, \boldsymbol{v}, \boldsymbol{w})=I_{+}+I_{-}+\alpha G+\beta U+\gamma V
$$

where

$$
\begin{aligned}
& I_{+}=\int_{\boldsymbol{\Omega}}\left(g(\boldsymbol{x})-f^{+}(\boldsymbol{x}-\boldsymbol{w})\right)^{2} d \boldsymbol{x}, I_{-}=\int_{\boldsymbol{\Omega}}\left(g(\boldsymbol{x})-f^{-}(\boldsymbol{x}+\boldsymbol{v})\right)^{2} d \boldsymbol{x} \\
& G=\int_{\boldsymbol{\Omega}}|\nabla g|^{2} d \boldsymbol{x}, U=\int_{\boldsymbol{\Omega}}\left(|\nabla \boldsymbol{v}|^{2}+|\nabla \boldsymbol{w}|^{2}\right) d \boldsymbol{x}, \quad V=|\boldsymbol{v}+\boldsymbol{w}-\boldsymbol{u}|^{2}
\end{aligned}
$$

Figure 2 shows the relations among $\boldsymbol{u}, \boldsymbol{v}, \boldsymbol{w}$ and $g$ and computational results of them.

Using the subframe optical flow, we extract a segment corresponding to the dominant plane $D_{H}$ using Algorithm 2. In Algorithm 2 for a pair of successive images $f_{i}$ and $f_{i+1}, \boldsymbol{u}_{i}$ is the optical flow field between $f_{i}$ and $f_{i+1}$, and $\boldsymbol{v}_{i}$ and $\boldsymbol{w}_{i}$ are the subframe optical flow fields between $f_{i}$ and $g$, and between $g$ and

\footnotetext{
${ }^{1}$ From eqs. (11) and (3), we have the relation $\nabla f(\boldsymbol{x}, t)^{\top}(\boldsymbol{v}+\boldsymbol{w})+f_{t} \cong 0$, since $f_{t}(\cdot, t+$ $\left.\frac{1}{2}\right)=f(\boldsymbol{x}, t+1)-f\left(\boldsymbol{x}, t+\frac{1}{2}\right)$ and $f_{t}(\cdot, t+1)=f\left(\boldsymbol{x}, t+1 \frac{1}{2}\right)-f(\boldsymbol{x}, t)$.
} 
$f_{i+1}$, respectively, where $g$ is the interframe image. Furthermore, the procedure Compute InterFrame $\left(f_{i}, f_{i+1}, \boldsymbol{u}_{i}, \alpha, \beta, \gamma\right)$ computes $g_{i}, \boldsymbol{v}_{i}$ and $\boldsymbol{w}_{i}$ from $f_{i}, f_{i+1}$ and $\boldsymbol{u}_{i}$ using the method proposed in the previous section. Moreover, in the algorithm $\boldsymbol{u}_{i}$ is computed from $f_{i}$ and $f_{i+1}$ using the large-displacement optical flow computation technique in [13].

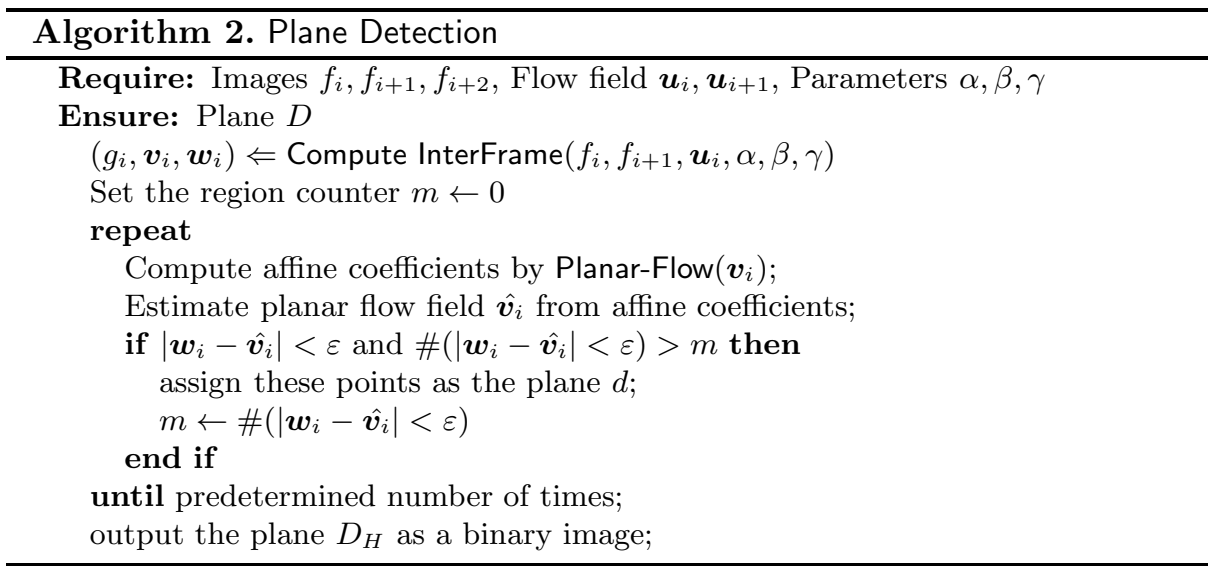

From $J(g, \boldsymbol{v}, \boldsymbol{w})$, for $g, \boldsymbol{v}$ and $\boldsymbol{w}$, we have the system of partial differential equations

$$
\Delta g-\frac{1}{\beta} F(g, \boldsymbol{v}, \boldsymbol{w})=0, \Delta \boldsymbol{v}-\frac{1}{\alpha} G^{-}(g, \boldsymbol{u}, \boldsymbol{w})=0, \Delta \boldsymbol{w}-\frac{1}{\alpha} G^{+}(g, \boldsymbol{u}, \boldsymbol{w})=0,
$$

where

$$
\begin{aligned}
F(g, \boldsymbol{v}, \boldsymbol{w}) & =2 g(\boldsymbol{x})-\left(f^{-}(\boldsymbol{x}+\boldsymbol{v})+f^{+}(\boldsymbol{x}-\boldsymbol{w})\right), \\
G^{-}(g, \boldsymbol{v}, \boldsymbol{w}) & =\gamma(\boldsymbol{v}+\boldsymbol{w}-\boldsymbol{u})+\left(f^{-}(\boldsymbol{x}+\boldsymbol{v})-g(\boldsymbol{x})\right) \nabla f^{-}(\boldsymbol{x}+\boldsymbol{v}), \\
G^{+}(g, \boldsymbol{u}, \boldsymbol{w}) & =\gamma(\boldsymbol{v}+\boldsymbol{w}-\boldsymbol{u})+\left(g(\boldsymbol{x})-f^{+}(\boldsymbol{x}-\boldsymbol{w})\right) \nabla f^{+}(\boldsymbol{x}-\boldsymbol{w}) .
\end{aligned}
$$

The mimimisation of $J(g, \boldsymbol{v}, \boldsymbol{w})$ is achieved by numerically solving eq. (7). Using semi-implicit discretisation of the associated diffusion equations such that

$$
\begin{aligned}
\partial_{t} g & =\Delta g-\frac{1}{\beta} F(g, \boldsymbol{v}, \boldsymbol{w}), \\
\partial_{t} \boldsymbol{v} & =\Delta \boldsymbol{v}-\frac{1}{\alpha} G^{-}(g, \boldsymbol{u}, \boldsymbol{w}), \\
\partial_{t} \boldsymbol{w} & =\Delta \boldsymbol{w}-\frac{1}{\alpha} G^{+}(g, \boldsymbol{u}, \boldsymbol{w}),
\end{aligned}
$$


we solve the system of iteration forms [12]

$$
\begin{gathered}
\frac{g^{(n+1)}-g^{(n)}}{\tau}=\Delta g^{(n+1)}-\frac{1}{\beta} F\left(g^{(n)}, \boldsymbol{v}^{(n)}, \boldsymbol{w}^{(n)}\right), \\
\frac{\boldsymbol{v}^{(n+1)}-\boldsymbol{v}^{(n)}}{\tau}=\Delta \boldsymbol{v}^{(n+1)}-\frac{1}{\alpha} G^{-}\left(g^{(n)}, \boldsymbol{u}^{(n)}, \boldsymbol{w}^{(n)}\right), \\
\frac{\boldsymbol{w}^{(n+1)}-\boldsymbol{w}^{(n)}}{\tau}=\Delta \boldsymbol{w}^{(n+1)}-\frac{1}{\alpha} G^{+}\left(g^{(n)}, \boldsymbol{u}^{(n)}, \boldsymbol{w}^{(n)}\right) .
\end{gathered}
$$

\section{Numerical Examples}

In experiments, we compared the statistics of the optical flow field computed by our method and by the pyramid-based Horn-Schunck method [8]. On the ground plane which corresponds to the dominant plane, since the optical flow field is smooth, we adopt the $L_{2}$ regularisation terms, that is, the regularisation term of the Horn-Schunck method is the square of the Frobenius norm $\operatorname{tr} \nabla \boldsymbol{u} \nabla \boldsymbol{u}^{\top}$ of the vector gradient $\nabla \boldsymbol{u}$ of the optical flow field $\boldsymbol{u}$.

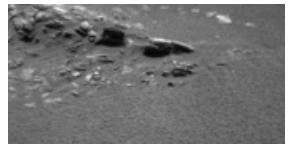

(a) sol13

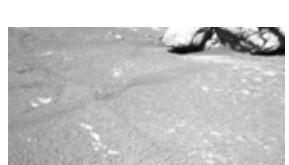

(d) $\operatorname{sol} 250$

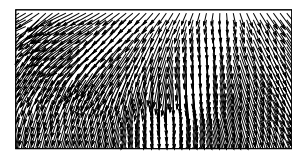

(b) Flow HS $\boldsymbol{u}$

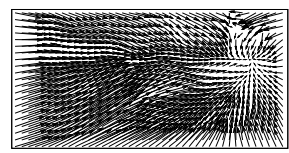

(e) Flow HS $\boldsymbol{u}$

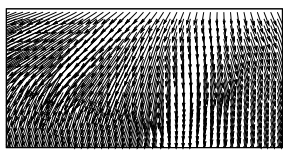

(c) Flow LDOF $\boldsymbol{u}$

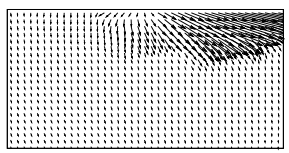

(f) Flow LDOF $\boldsymbol{u}$

Fig. 3. Images and optical flows of Sol13 and Sol250. (a) Sol13. (b) Optical flow of Sol13 computed by the Horn-Schanck method with pyramid transform 9]. (c) Optical flow of Sol13 computed by the large-displacement method [13]. (d) Sol250. (e) Optical flow of Sol250 computed by the Horn-Schanck method with the pyramid transform [9]. (f) Optical flow of Sol250 computed by the large-displacement method [13].

Figure 3 shows images and their optical flow fields computed by two methods. The top and bottom rows show images and optical flow fields of Sol13 and Sol205, respectively. (b) and (e) show optical flow fields of Sol13 and Sol250, respectively, computed by the Horn-Schanck method [8] with pyramid transform (HSP). (c) and (f) show optical flow fields of Sol13 and Sol 250, respectively, computed by the Large-Displacement Optical Flow method [13] (LDOF). 


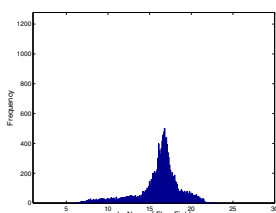

(a) $\operatorname{HSP} \boldsymbol{u}$

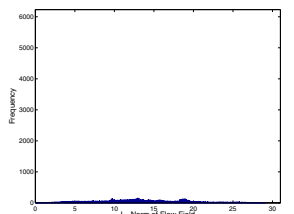

(e) HSP $\boldsymbol{u} \operatorname{Sol} 250$

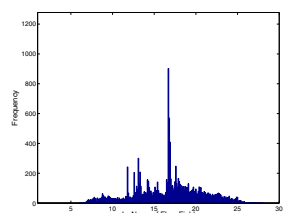

(b) LDOF $\boldsymbol{u}$ Sol13

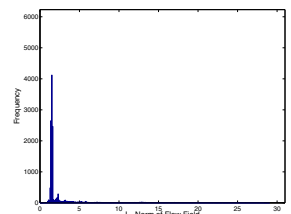

(f) LDOF $\boldsymbol{u} \operatorname{Sol} 250$

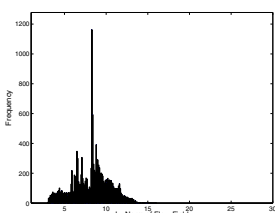

(c) TOSR $\boldsymbol{v}$ Sol13

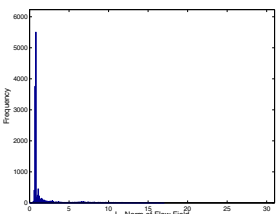

(g) TOSR $v$ Sol250

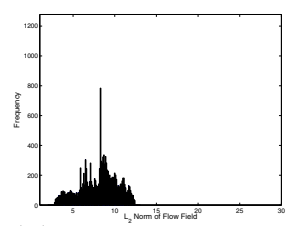

(d) TOSR $\boldsymbol{w}$ Sol13

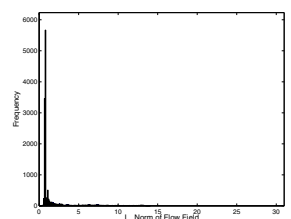

(h) TOSR $\boldsymbol{w} \operatorname{Sol} 250$

Fig. 4. Norm Histogram of Sol13 and Sol250. The top and bottom rows shows results for Sol13 and Sol250, respectively. From left to right, the histograms of $\boldsymbol{u}$ computed by the Horn-Schunck method with pyramid transform, $\boldsymbol{u}$ computed by the largedisplacement optical flow method, $\boldsymbol{v}$ and $\boldsymbol{w}$, which are computed by our method -the temporal optical flow superresolurion, respectively.

Table 1. Kurtosis of histograms

\begin{tabular}{|c||c|c|c|c|}
\hline & HS $\boldsymbol{u}$ & LDOF $\boldsymbol{u}$ & TOSR $\boldsymbol{v}$ & TOSR $\boldsymbol{w}$ \\
\hline Sol13 & 7.04 & 50.33 & 33.66 & 15.82 \\
\hline Sol250 & 2.71 & 115.51 & 92.75 & 81.63 \\
\hline
\end{tabular}

Figure 4 shows the histograms for the $l_{2}$-norms of the optical flow vectors for the results of Fig. 3. In Fig. 4, the top and bottom rows show results for Sol13 and Sol250, respectively. Furthermore, from left to right, the histograms of $\boldsymbol{u}$ computed by the HSP method, $\boldsymbol{u}$ computed by the LDOF method, $\boldsymbol{v}$ and $\boldsymbol{w}$, which are computed by our flow-field up-conversion based on the Temporal Optical flow Superresolution (TOSR).

Figure 5 shows the second time derivatives of the histograms, since the second time derivatives of distributions allow to detect peaks in the distributions. Table 1 shows the kurtoses of optical flow fields computed by the HSP method, the LDOF method and TOSR method. Kurtoses of optical flow field computed by

Table 2. Unification of dominant plane segments by three methods for Sol13 and Sol250. The entries are $\left|D_{\alpha}\right| /\left|D_{k}\right|$ for $\alpha\{H, B, P\}$ and $k=1,2,3$.

\begin{tabular}{|c|c|c|c|c||c|c|c|c|c|}
\hline Sol13 & $D_{B}$ & $D_{P}$ & $D_{H}$ & average & Sol250 & 1.00 & 0.80 & 0.80 & 0.87 \\
\hline$D_{1}$ & 0.99 & 0.34 & 0.51 & 0.61 & $D_{1}$ & 1.00 & 0.80 & 0.80 & 0.8 \\
\hline$D_{2}$ & 0.63 & 0.71 & 0.88 & 0.74 & $D_{2}$ & 0.82 & 0.98 & 0.99 & 0.93 \\
\hline$D_{3}$ & 0.23 & 0.87 & 0.71 & 0.60 & $D_{3}$ & 0.78 & 0.99 & 0.98 & 0.92 \\
\hline
\end{tabular}




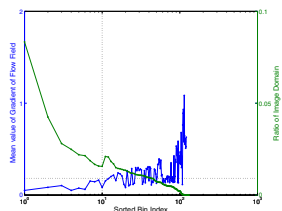

(a) $h_{t t} \boldsymbol{v}$ Sol13

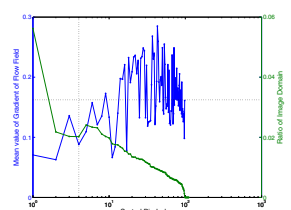

(b) $h_{t t} \boldsymbol{w}$ Sol13

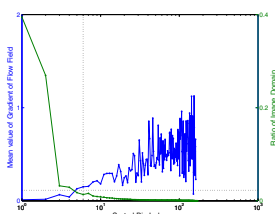

(c) $h_{t t} \boldsymbol{v}$ Sol250

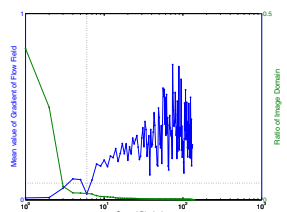

(d) $h_{t t} \boldsymbol{w}$ Sol250

Fig. 5. Second Time derivatives of the histograms of Sol13 and Sol250. The vertical line in each figure show the peak of the histogram. The horizontal line in each figure corresponds to the total variation of the optical flow vectors $\int_{\Omega}|\nabla \boldsymbol{u}| d \boldsymbol{x}$. The green curve in each figure shows the ration $|\Omega(k)| /|\Omega|$, where $\Omega(k)$ is the region corresponding to the $k$-th bin in the histogram.

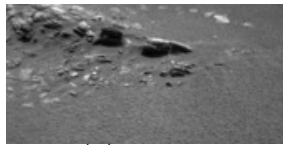

(a) sol13

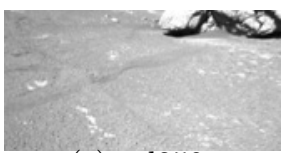

(e) $\operatorname{sol} 250$

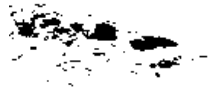

(b) Binarisation

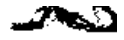

(f) Binarisation

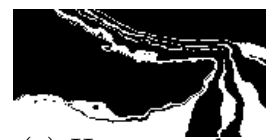

(c) Homogarphy

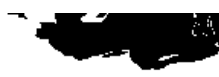

(g) Homography (d) Peak-based

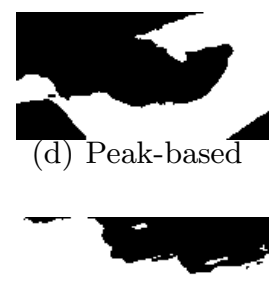

(h) Peak-based

Fig. 6. Results for Sol13 and Sol250. The top and bottom rows show results for Sol13 and Sol250, respectively. From left to right, original images and results computed by image binarisation, the peak detection and the homograpy-based method, are shown.

the LDOF and TOSR methods are both larger than those by the HSP method. In each frame, the histograms of the norm of the optical flow vectors computed by the HSP method are unimodal with wide divergence. Therefore, we cannot detect the dominant plane from the optical field. The histograms of the norm of the optical flow vectors computed by our method are, however, multimodal or with small divergence. If semantic planar segments exist in a frame, the optical flow vectors are stationary on this region. Then, the peaks appear in the histogram of the norm of th optical flow vectors. Comparison of the optical flow fields by two methods leads to the conclusion that the LDOF method can detect these peaks and that the HSP method fails to detect these peaks in both examples.

However, since as described in the previous sections, for the semantic segmentation of the dominant plane as a safe area in the workspace, we are required to have three successive frames of images. Therefore, we generate two successive optical flow fields from a pair of successive images using the temporal superresolution of the optical flow field. As shown in Fig. 4, we can extract a semantic segment using statistical bias of the optical flow vectors on the dominant plane. 


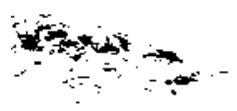

(a) 1 method

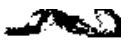

(d) 1 method

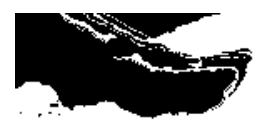

(b) 2 methods

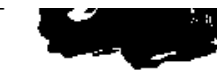

(e) 2 methods

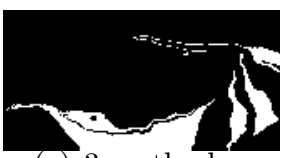

(c) 3 methods

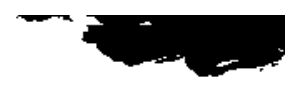

(f) 3 methods

Fig. 7. Unification of the results obtained by three methods. The top and bottom row are results for sol13 and sol250, respectively. From left to right, the unification results for one-, tow- and three methods, respectively.

Setting $h(\boldsymbol{u}(\boldsymbol{x}), t)$ for $\boldsymbol{x}=(x, y)^{\top}$ to be histogram of $|\boldsymbol{u}(\boldsymbol{x})|$, the peaks $t$ in the histogram are bins which satisfy the relations $h(\boldsymbol{u}(\boldsymbol{x}), t)>$ 2average $_{t} h(\boldsymbol{u}(\boldsymbol{x}))$ and $h(\boldsymbol{u}(\boldsymbol{x}), t)>$ 2average $_{t} h_{t t}(\boldsymbol{u}(\boldsymbol{x}))$. The peak-based segmentation detects the region $D_{P}=\{\boldsymbol{x} \mid t \in T$ for $h(\boldsymbol{u}(\boldsymbol{x}), t)\}$. Furthermore, we extract a segment $D_{B}=$ $\{\boldsymbol{x} \mid f(x, y, t)>A\}$ by binarising grey-values of images using $A$ computed by the Otsu threshold method [15].

Figure 6 shows the results. For the comparison, we also computed semantic segments using $D_{P}$ and $D_{B}$. These results show that our proposing method, which combines the homgraphy and temporal optical flow superrealution, achieves semantic segmentation from a video sequence for navigation. Table 2 and Figure 7 show the results of the unification of the results obtained by three methods. Using the Boolean functions

$$
f_{H}(\boldsymbol{x})=\left\{\begin{array}{l}
1, \text { if } \boldsymbol{x} \in D_{H} \\
0, \text { otherwise, }
\end{array} \quad f_{B}(\boldsymbol{x})=\left\{\begin{array}{l}
1, \text { if } \boldsymbol{x} \in D_{B} \\
0, \text { otherwise, }
\end{array} \quad f_{P}(\boldsymbol{x})=\left\{\begin{array}{l}
1, \text { if } \boldsymbol{x} \in D_{P} \\
0, \text { otherwise }
\end{array}\right.\right.\right.
$$

we define the three regions,

$$
\begin{aligned}
& D_{1}=\left\{\boldsymbol{x} \mid f_{H}(\boldsymbol{x}) \vee f_{B}(\boldsymbol{x}) \vee f_{P}(\boldsymbol{x})=1\right\}, \\
& D_{2}=\left\{\left(f_{H}\left(\boldsymbol{x} \wedge f_{B}(\boldsymbol{x})\right) \vee\left(f_{B}(\boldsymbol{x}) \wedge f_{P}(\boldsymbol{x})\right) \vee\left(f(\boldsymbol{x}) \wedge f_{H}(\boldsymbol{x})\right)=1\right\},\right. \\
& D_{3}=\left\{f_{H}(\boldsymbol{x}) \wedge f_{B}(\boldsymbol{x}) \wedge f_{P}(\boldsymbol{x})=1\right\} .
\end{aligned}
$$

For $k=1,2,3, D_{k}$ is the collection of pixels which are categorised as elements of dominant plane by the $k$ different algorithms. Then, we evaluate $\left|D_{\alpha}\right| /\left|D_{k}\right|$ for $\alpha\{H, B, P\}$ and $k=1,2,3$, where $|D|$ is the area of region $D$, since the ground truths are not prepared for these image sequences. In Fig. (17), the top and bottom row are results for sol13 and sol250, respectively, and From left to right, the unification results for one-, tow- and three methods, respectively, are shown. These results show that unification of the three methods improves the results. 


\section{Conclusions}

In this paper, we introduced a method for semantic segmentation of the dominant plane on an image sequence from a low-frame rate optical flow field.

In refs. [1] and [10], a dominant plane detection was achieved from a triplet of successive images. We have proposed a method for the detection of the dominant plane from low-frame rate image sequences using sub-frame optical flow computation.

\section{References}

1. Ohnishi, N., Imiya, A.: Featureless robot navigation using optical flow. Connection Science 17, 23-46 (2005)

2. Braillon, C., Pradalier, C., Crowley, J.L., Laugier, C.: Real-time moving obstacle detection using optical flow models. Intelligent Vehicles Symposium, 466-471 (2006)

3. Liang, B., Pears, N.: Visual navigation using planar homographies. In: IEEE International Conference on Robotics and Automation, pp. 205-210 (2002)

4. Young-Geun, K., Hakil, K.: Layered ground floor detection for vision-based mobile robot navigation. In: International Conference on Robotics and Automation, pp. 13-18 (2004)

5. Fischer, B., Modersitzki, J.: Ill-posed medicine- an introduction to image registration. Inverse Problem 24, 1-17 (2008)

6. Vardy, A., Moller, R.: Biologically plausible visual homing methods based on optical flow techniques. Connection Science 17, 47-89 (2005)

7. Beauchemin, S.S., Barron, J.L.: The computation of optical flow. ACM Computer Surveys 26, 433-467 (1995)

8. Horn, B.K.P., Schunck, B.G.: Determining optical flow. Artificial Intelligence 17, 185-204 (1991)

9. Hwang, S.-H., Lee, U.K.: A hierarchical optical flow estimation algorithm based on the interlevel motion smoothness constraint. Pattern Recognition 26, 939-952 (1993)

10. Ohnishi, N., Imiya, A.: Dominant plane detection from optical flow for robot navigation. Pattern Recognition Letters 27, 1009-1021 (2006)

11. Fischler, M.A., Bolles, R.C.: Random sample consensus: A paradigm for model fitting with applications to image analysis and automated cartography. Comm. of the ACM 24, 381-395 (1991)

12. Varga, R.S.: Matrix Iteration Analysis, 2nd edn. Springer (2000)

13. Brox, T., Malik, J.: Large displacement optical flow: descriptor matching in variational motion estimation. IEEE Trans. PAMI 33, 500-513 (2011)

14. Hartley, R., Zisserman, A.: Multiple View Geometry in Computer Vision. Cambridge University Press (2000)

15. Otsu, N.: A threshold selection method from gray-level histograms. IEEE Trans. Sys., Man., Cyber. 9, 62-66 (1979) 